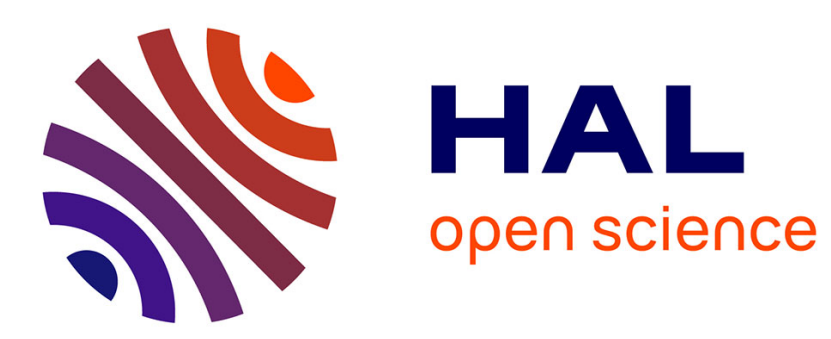

\title{
Trailing Edge Noise Computation of a Fan Blade Profile
} Julien Christophe, Stéphane Moreau, Jérome Anthoine

\section{To cite this version:}

Julien Christophe, Stéphane Moreau, Jérome Anthoine. Trailing Edge Noise Computation of a Fan Blade Profile. 10ème Congrès Français d'Acoustique, Apr 2010, Lyon, France. hal-00541709

\section{HAL Id: hal-00541709 \\ https://hal.science/hal-00541709}

Submitted on 1 Dec 2010

HAL is a multi-disciplinary open access archive for the deposit and dissemination of scientific research documents, whether they are published or not. The documents may come from teaching and research institutions in France or abroad, or from public or private research centers.
L'archive ouverte pluridisciplinaire HAL, est destinée au dépôt et à la diffusion de documents scientifiques de niveau recherche, publiés ou non, émanant des établissements d'enseignement et de recherche français ou étrangers, des laboratoires publics ou privés. 


\title{
10ème Congrès Français d'Acoustique
}

Lyon, 12-16 Avril 2010

\section{Trailing Edge Noise Computation of a Fan Blade Profile}

\author{
Julien Christophe ${ }^{1}$, Stéphane Moreau², Jérome Anthoine ${ }^{2}$ \\ 1 von Karman Institute for Fluid Dynamics, 72 Chaussée de Waterloo, 1640 Rhode-St-Genèse, Belgium, julien.christophe@vki.ac.be \\ ${ }^{2}$ Université de Sherbrooke, 2500 Boulevard de l'Université, Sherbrooke, J1K 2R1 Québec, Canada, Stephane.Moreau@USherbrooke.ca \\ ${ }^{3}$ ONERA - The French Aerospace Lab, Midi-Pyrenees Center, Mauzac, France, Jerome.Anthoine@onera.fr
}

In problem involving noise generated by fans or high-lift devices in uniform stationary flow, trailing edge noise has a primary interest. This paper proposes to study the trailing edge noise produced by a Controlled-Diffusion (CD) airfoil specially developed for automotive engine cooling by Valeo. LES flow computations are realised using the open source OpenFOAM solver on different grids for a Reynolds number based on the chord of $R e_{C}=1.6 \times 10^{5}$ and an angle of attack of 8 degrees. The computations are compared with pressure and velocity measurements obtained respectively by both pressure probes and hot-wire anemometry. The results show the necessity of selecting proper boundary conditions and grid refinement in order to obtain correct flow prediction around the trailing edge. The far field noise is computed using the Ffowcs-Williams and Hall's anology using velocity information in a volume around the trailing edge and is compared to microphone measurements. The different mechanisms of sound production appearing at different frequencies are highlighted.

\section{Introduction}

Trailing-edge noise or broadband self-noise, caused by the scattering of boundary-layer vortical disturbances into acoustic waves, occurs at the trailing edge of any lifting surface. It can even become the dominant source of noise generated by rotating machines such as fans, turboengines [1], wind turbines [2], and other high-lift devices [3] in the absence of any other interaction noise source. These noise levels can be hopefully reduced by properly identifying the sources of self-noise, and subsequently modifying design parameters that affect these noise sources. The large computational costs associated with unsteady turbulent flow simulations have however limited most numerical studies to simplified geometries such as airfoils. The first LES of the flow over the Valeo Controlled-Diffusion (CD) airfoil was performed by Wang [4] at a moderate angle of attack (a.o.a) of $8^{\circ}$, which corresponds to the design condition or the maximum static efficiency of the associated fan. It used a large structured mesh with 5.1 million nodes to yield stable and accurate flow solutions. Subsequent attempts to reduce the computational costs included the use of non-conforming boundary methods such as the Lattice Boltzmann method and Immersed Boundary method [5], as well as hybrid solution methods such Detached Eddy Simulations [6]. These techniques yielded less accurate mean wall-pressure distributions (larger laminar recirculation bubble near the leading edge and possibly turbulent flow separaration near the trailing edge) and frequency spectra near the trailing edge than those obtained by Wang [4]. An alternative approach was then taken by Moreau et al. [7], involving the flow solver CDP and several LES on unstructured grid topologies. It showed that accurate results for all flow quantities could be obtained with the unstructured flow solver, provided the grid remained smooth and regular enough in all directions.

The present work proposes to study the influence of mesh refinement on the LES flow for this moderate angle of attack $\left(\alpha_{w}=8^{\circ}\right)$. This will help to define the methodology to apply and possibly improvements required in such airfoil flow conditions to reach reliable results compared to experiments. This study encompasses the correct resolution of the flow around the airfoil and the prediction of the trailing edge noise using the Ffowcs-Williams and Hall's analogy, specially developped for trailing edge noise.

\section{Experimental database}

The first experimental data, including wall-pressure measurements and some of the hot-wire measurements, were collected in the large anechoic wind tunnel of the Ecole Centrale de Lyon (ECL). Additional hot-wire measurements have been conducted at Michigan State University (MSU), where the MSU $0.61 \mathrm{~m} 2$ tunnel has been modified to closely match the configuration of the ECL wind tunnel.

The airfoil mock-up has a $13.4 \mathrm{~cm}$ constant chord length $(C)$ and a $0.3 \mathrm{~m}$ span $(L)$. It is held between two horizontal side plates fixed to the nozzle of the open-jet wind tunnel. These plates are $25 \mathrm{~cm}(\approx 1.85 C)$ apart and the width of the rectangular jet is $50 \mathrm{~cm}(\approx 3.7 C)$. All of the tests presented here were run with a speed $U_{0}=16 \mathrm{~m} / \mathrm{s}$, which corresponds to a Reynolds number based on the airfoil chord length $R e_{C}=1.6 \times 10^{5}$ and an angle of attack with respect to the chord, $\alpha_{w}$, of $8^{\circ}$.

The CD airfoil mock-up is equipped at midspan with 21 flush-mounted remote Electret microphone probes (RMP) (Pérennès \& Roger [3]). The RMPs measure 


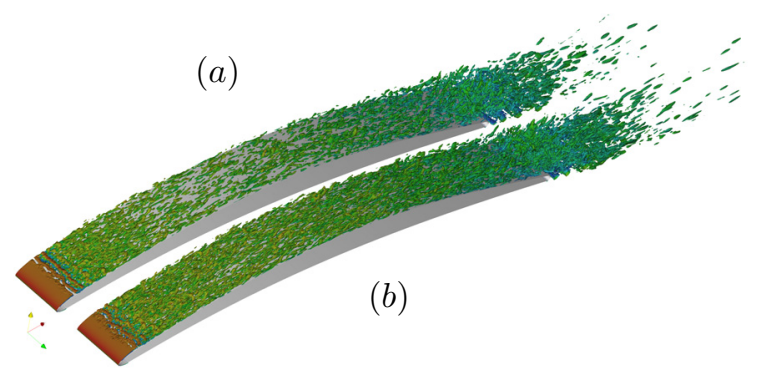

Figure 1: Flow topology described by the $Q$ factor ( $Q=1000 s^{-2}$ iso-contours) for both computations :

(a) 64 spanwise cells and (b) 128 spanwise cells.

both the mean and fluctuating pressure within a frequency range of $20 \mathrm{~Hz}-25 \mathrm{kHz}$. Details of the wallpressure measurements in this configuration can be found in Moreau \& Roger [8]. The far-field noise is measured simultaneously using a single B\&K $1.27 \mathrm{~cm}\left(1 / 2^{\prime}\right)$ Type-4181 microphone.

\section{LES flow computations}

\subsection{Flow solver}

The LES is based on the spatially filtered, incompressible Navier-Stokes equations with the dynamic subgridscale model. Equations are solved with the open source finite volume solver OpenFOAM using schemes that are second-order accurate in space and time. Preliminary RANS computations are required to provide the LES boundary conditions for computations, as described in the next section. These are performed using the ShearStress-Transport (SST) $k-\omega$ turbulence model, with again second order accurate solution for all variables.

\subsection{Grid topologies and boundary con- ditions}

The flow around an airfoil in an open-jet wind tunnel facility differs significantly from that around an isolated airfoil in a uniform stream. In the present case, the airfoil is immersed in a jet of finite width, which is deflected by the circulation created by the airfoil and then has an impact on the airfoil loading and the corresponding noise created. This can be seen by the large deflection of the bounding shear layers (Moreau et al. [9]). In order to match experiments closely with the LES computations, the following procedure is used. A 2-D ReynoldsAveraged Navier-Stokes (RANS) simulation of the complete open-jet wind tunnel configuration including the nozzle, the airfoil and part of the anechoic chamber is first required to capture the strong interaction between the jet and the CD airfoil and its impact on the airfoil load at any incidence. The full RANS simulation provides velocity boundary conditions for a smaller LES truncated domain, which is embedded in the potential core of the jet. This method proved its efficiency, as the first LES of the CD airfoil at a small angle of attack of $8^{\circ}$ performed by Wang [4] closely reproduced the experimental conditions found in the ECL large anechoic wind tunnel.
The low Reynolds number $R e_{C}$ and the large jetwidth to chord ratio in the present experiment allows a computational domain around the full airfoil. The size of the computational domain is : $4 C$ in the streamwise $(x)$ direction, $2.5 C$ in the crosswise $(y)$ direction and $0.1 C$ in the spanwise $(z)$ direction. The resulting LES grid is a single block-structured C-mesh, with $960 \times 84$ $\times N$ cells, where $N$ can be 64 or 128 , in the present computations. Smooth grid-distribution and orthogonality at the wall are again applied and the grid-stretching ratio is limited in the streamwise and crossflow directions to ensure numerical stability.

The LES use a no-slip boundary condition on the airfoil surface, a convective outflow boundary condition at the exit plane, and the steady RANS velocity ( $U$ and $V$ ) along the upper and lower boundaries. Periodic boundary conditions are applied in the spanwise direction.

Computations were run for at least 5 flow-through times, based on the freestream velocity and airfoil chord length, before a statistically steady state was reached and mean values were collected. Airfoil surface pressure and wake velocity statistics were then acquired for a period of at least 4 flow-through with a sampling rate of $50 \mathrm{kHz}$.

\subsection{Results}

The flow topology is first illustrated in Fig. 1 by the isocontours of constant $Q$ factor, which show the level of vorticity and the size of the turbulent structures in the flow at a given instant. It shows for both cases a laminar boundary layer on the lower (pressure) side of the airfoil, and a transitional and turbulent boundary layer on the upper (suction) side. Transition on the suction side is trigerred by an unsteady laminar separation near the leading edge, where small vortices are born close to the reattachment point of the laminar recirculation bubble. The flow re-laminarizes towards mid-chord because of the favorable pressure gradient. When this gradient becomes adverse, the boundary layer thickens again and more and larger vortices are created towards the trailing edge. Like its laminar counterpart on the pressure side, the turbulent boundary layer remains attached as it passes the trailing edge despite of the strong adverse gradient. Furthermore, the influence of the mesh refinement in the spanwise direction is clearly observed in Fig. 1. As expected, smaller structures are formed directly after the recirculation bubble with 128 cells in the spanwise direction instead of 64 . These structures are then convected along the airfoil chord, resulting in a wake containing more structures with a finer grid. In the recirculation region, no speficic differences are observed with the mesh refinement. Moreover, vortex shedding seems to appear from the pressure side at the trailing edge, as observed in the experiments. The importance of this phenomenon on the noise radiation will be illustrated in section 4 .

The mean and fluctuating wall-pressure were analyzed for each of the LES runs and compared with the RMPs measurements by Moreau \& Roger [8]. The mean pressure on the surface, characterized by the pressure coefficient $-C_{p}$, is shown in Fig. 2.

These results show that the LES computations are in 


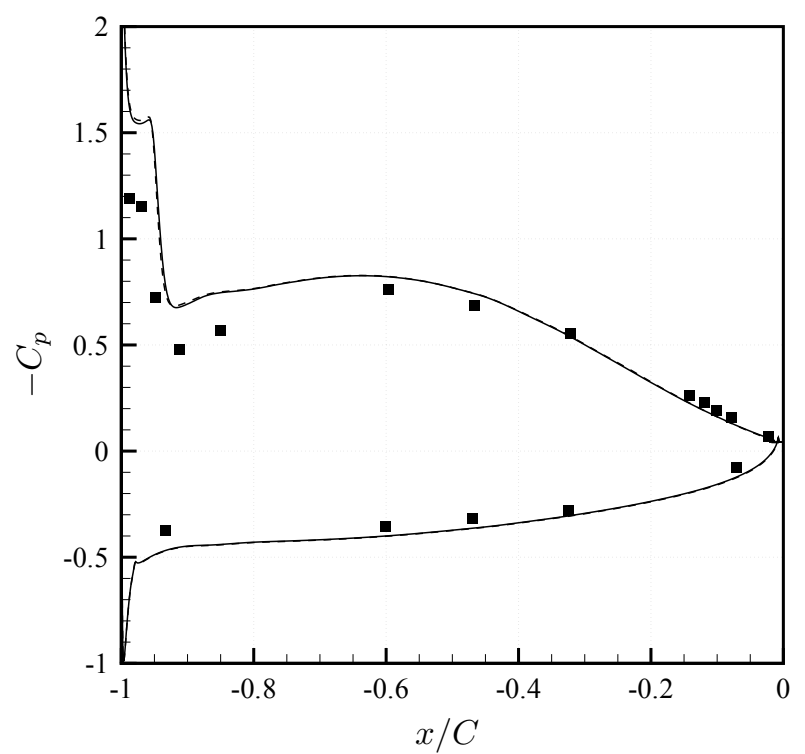

Figure 2: Mean wall-pressure coefficient $-C_{p}$ along all blade surface for both computations : (plain) 64 spanwise cells and (dash) 128 spanwise cells.

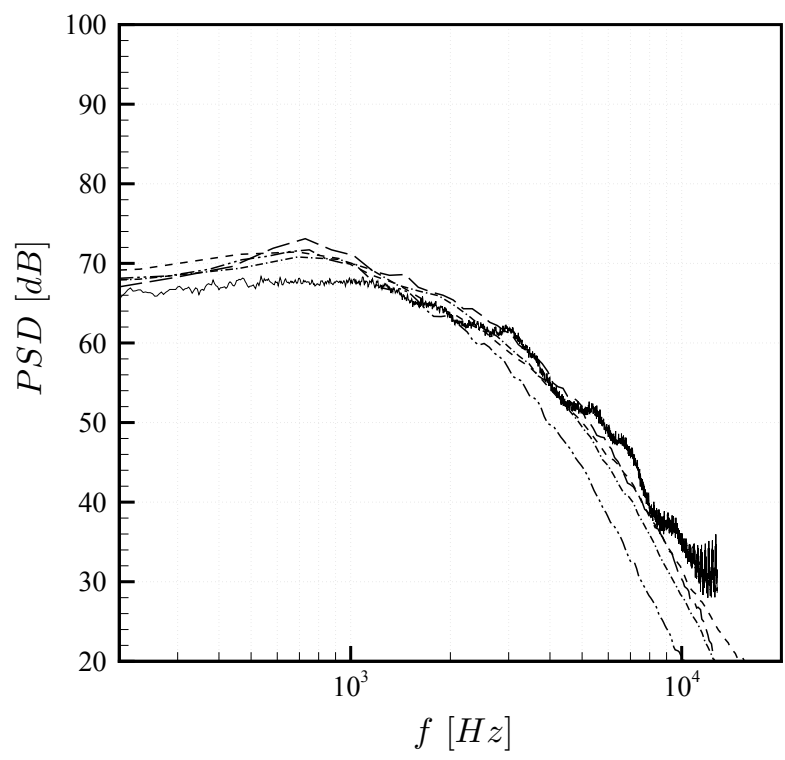

Figure 3: Frequency spectra of the wall pressure fluctuations on the suction side in the leading edge area $(x / C=-0.02):$ (dash) 64 spanwise cells,

(dash-dot) 128 spanwise cells, (dash-dot-dot) Wang's computation and (long dash) CDP computation.

(plain) Experiments.

reasonable good agreement with experiments. This also illustrates that the approach, consisting in transfer of flow boundary conditions between the RANS and LES computations, provides high fidelity in terms of global flow conditions. Only small differences are observed between the two present computations in the leading edge recirculation region.

The wall-pressure spectra near the trailing edge are compared with the measurements above RMP\#25 in Fig. 3 for both computations. Both LES results are very similar, particularly for $f>2000 \mathrm{~Hz}$, where all the numerical pressure spectra agree with the experimen-
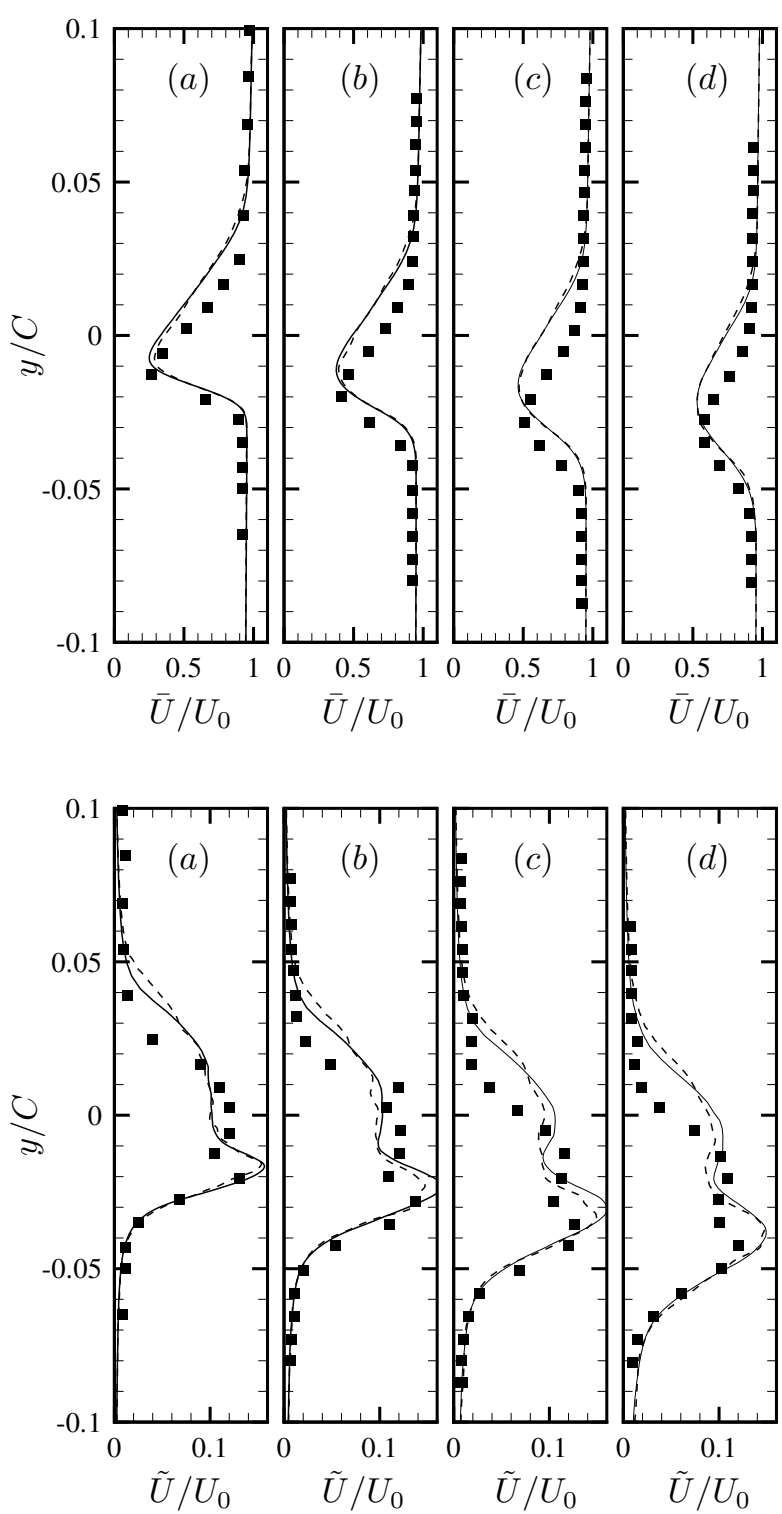

Figure 4: Wake velocity data. (top) Normalized mean velocity $\bar{U} / U_{0}$ and (bottom) Normalized rms velocity

fluctuations $\tilde{U} / U_{0}$ at four $x / C$ locations : (a) $x / C=0.0574$, (b) $x / C=0.0940$, (c) $x / C=0.1313$ and (d) $x / C=0.1686$. (plain) 64 spanwise cells and

(dash) 128 spanwise cells. (dots) Experiments.

tal one. The main differences appear at low and mid frequencies where the effects of the mesh refinement is observed. The improvement in the mesh refinement is reducing the gap between the numerical and experimental pressure spectra at low frequencies (below $500 \mathrm{~Hz}$ ) up to $2 \mathrm{~dB}$ at the trailing edge. Similar behavior has been also observed in Christophe et al. [10]. Nevertheless, the best computation (128 spanwise cells) is still overpredicting the pressure spectrum at low and mid frequencies compared to experiments (up to $5 \mathrm{~dB}$ ). A comparison of the present computations with other computations in the same conditions on the identical grid, but using different codes, is also shown. The CDP and present results show better agreement with the experimental data at higher frequencies than those obtained by Wang [4], which tail off more quickly with frequencies, suggesting that boundary layers lacks very small 
scale structures. At low frequencies, the present computation predicts similar amplitude of the wall-pressure spectrum compared to other CFD results.

Velocity data were extracted for each computation, at locations that matched those of the hot-wire probes in the experiments of Moreau et al. [9]. Fig. 4 shows the mean and rms of velocity fluctuations for the streamwise $U$ component at four different measurement stations from $x / C=0.0574$ to $x / C=0.1686$. For all stations, and for both mesh refinements, computations present very similar shapes with a wake deficit close to those of the experiments. Nevetheless, a slight shift appeared systematically in the crosswise direction between the computations and the experiments, illustrated by an underestimation in the lower part of the wake (pressure side) and an overestimation in the upper part of the wake (suction side). Concerning the rms of the $x$ velocity fluctuations, both mesh refinements show similar profiles qualitatively than those observed in the experiments. Both computations reproduce correctly the position of the maximum peak of rms of the $x$-velocity correctly(the location of the higher shear in the wake). The amplitude of this peak is overpredicted by both computations for all stations. Finally, as for the velocity, the rms of the $x$-velocity is overpredicted in the upper part of the wake for both mesh refinements.

\section{Noise computation}

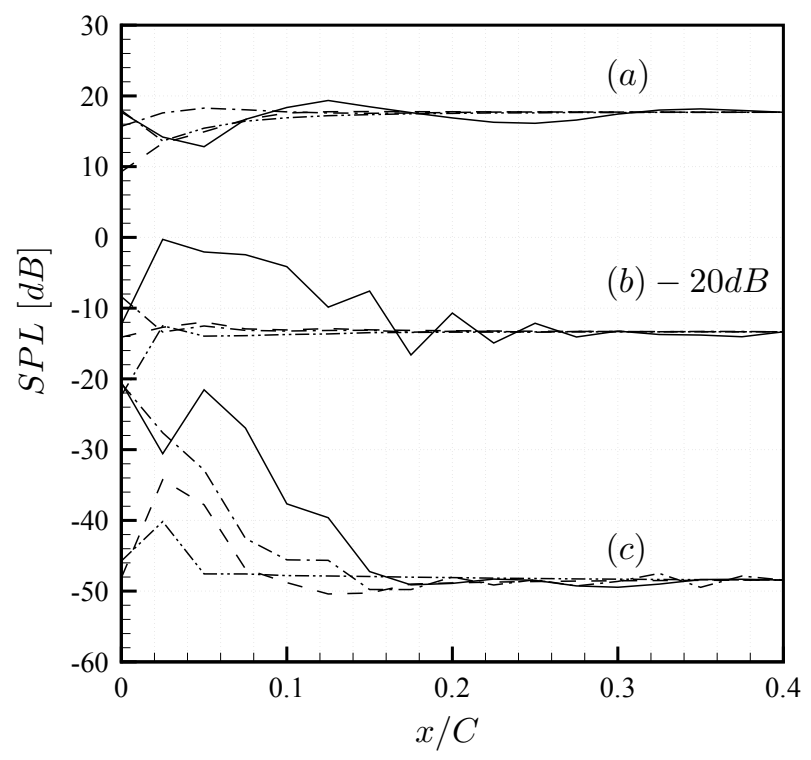

Figure 5: Evolution of the far field acoustic spectra in the mid span plane above the airfoil $\left(\theta=90^{\circ}\right.$,

$R=2 \mathrm{~m}$ ) with the distance of the source integration domain boundaries from the trailing edge for different frequencies : (a) $446.9 \mathrm{~Hz}$, (b) $1787.8 \mathrm{~Hz}(-20 \mathrm{~dB})$ and (c) $7151.2 \mathrm{~Hz}$. (plain) right, (dash) top, (dash-dot) left and (dash-dot-dot) bottom boundary.

\subsection{Ffowcs-Williams and Hall's analogy}

Similarly to other well know hybrid methods based on acoustic analogies [11, 12], the Ffowcs-Williams and
Hall's analogy is considering the decoupling of the sound sources computation (ie. the LES computation described above) and the sound computation, considered as a post-processing step of the source computation. Because the source field is computed from incompressible Navier-Stokes equations, a hard-wall Green's function, whose normal derivative vanishes on the airfoil surface, should be employed to provide a correct solution to the Lighthill equation. When the acoustic wavelength $\lambda$ is much longer than the thickness of the airfoil $h$ and much shorter than the chord $C(h \ll \lambda \ll C)$, the airfoil trailing-edge can be approximated by a semiinfinite plate and then, half-plane Green's function can be used to solve lighthill equation leading to the FfowcsWilliams and Hall's analogy. The far field acoustic pressure at a given observer location $\vec{x}(r, \theta, z)$ and for a given angular frequency $\omega$ :

$$
\begin{array}{r}
p_{a}^{\infty}(\vec{x}, \omega)=\frac{2 e^{-i(\pi / 4)}}{\pi^{\frac{1}{2}}} k^{2} \sin \frac{\theta}{2} \int_{V} \frac{e^{i k R}}{4 \pi R} \frac{(\sin \phi)^{\frac{1}{2}}}{\left(2 k r_{0}\right)^{\frac{1}{2}}} \\
\left\{\rho_{0}\left(\widehat{u_{\theta}^{2}}-\widehat{u_{r}^{2}}\right) \sin \frac{\theta_{0}}{2}-2 \rho_{0} \widehat{u_{r} u_{\theta}} \cos \frac{\theta_{0}}{2}\right\} d^{3} \vec{y}
\end{array}
$$

where the caret denotes the temporal fourier tranform and $V$ is the volume domain around the trailing edge in which the velocity components $U_{r}$ and $U_{\theta}$, defined in cylindrical-polar coordinates, are used. The vector $\vec{y}\left(r_{0}, \theta_{0}, z_{0}\right)$ represents the source-field points with $R=|\vec{x}-\vec{y}|$ and $\sin \phi=r /\left[r^{2}+\left(z-z_{0}\right)^{2}\right]^{\frac{1}{2}}$. The noise calculation can be simplified further if the spanwise extent of the source field is acoustically compact. This is generally not the case for the full span airfoil of the experiments but is a reasonnable assumption for the source region contained in the computational domain. The simplified formulation is then given as :

$$
p_{\infty}^{a}(\vec{x}, \omega)=\frac{e^{i(k|\vec{x}|-\pi / 4)}}{2^{\frac{5}{2}} \pi^{\frac{3}{2}}|\vec{x}|}(k \sin \phi)^{\frac{1}{2}} \sin \frac{\theta}{2} \widehat{S}(\omega)
$$

where

$$
S(t)=\int_{V} \frac{\rho_{0}}{r_{0}^{\frac{3}{2}}}\left\{\left(u_{\theta}^{2}-u_{r}^{2}\right) \sin \frac{\theta_{0}}{2}-2 u_{r} u_{\theta} \cos \frac{\theta_{0}}{2}\right\} d^{3} \vec{y}
$$

This second formulation is convenient to evaluate easily during the LES computation the single compact source term $\mathrm{S}(\mathrm{t})$.

\subsection{Results}

To compute the source terms in Eq. (3), the velocity components have been extracted in the complete computationnal domain every 10 time steps during the LES computation with 64 spanwise cells. The extraction period is $t U_{0} / C=4$ for a total record of $\mathrm{N}=2000$ time samples with resolution of $\Delta t U_{0} / C=0.002$. The total signal is divided in 9 segments with $50 \%$ overlap. The aperiodic time signal are multiplied by the Hanning window function and discrete Fourier transforms are performed. The resulting coefficients are renormalized such that the power spectrum computed from them, when integrated over all positive frequencies, gives the meansquare fluctuations of the original function. Each signal 

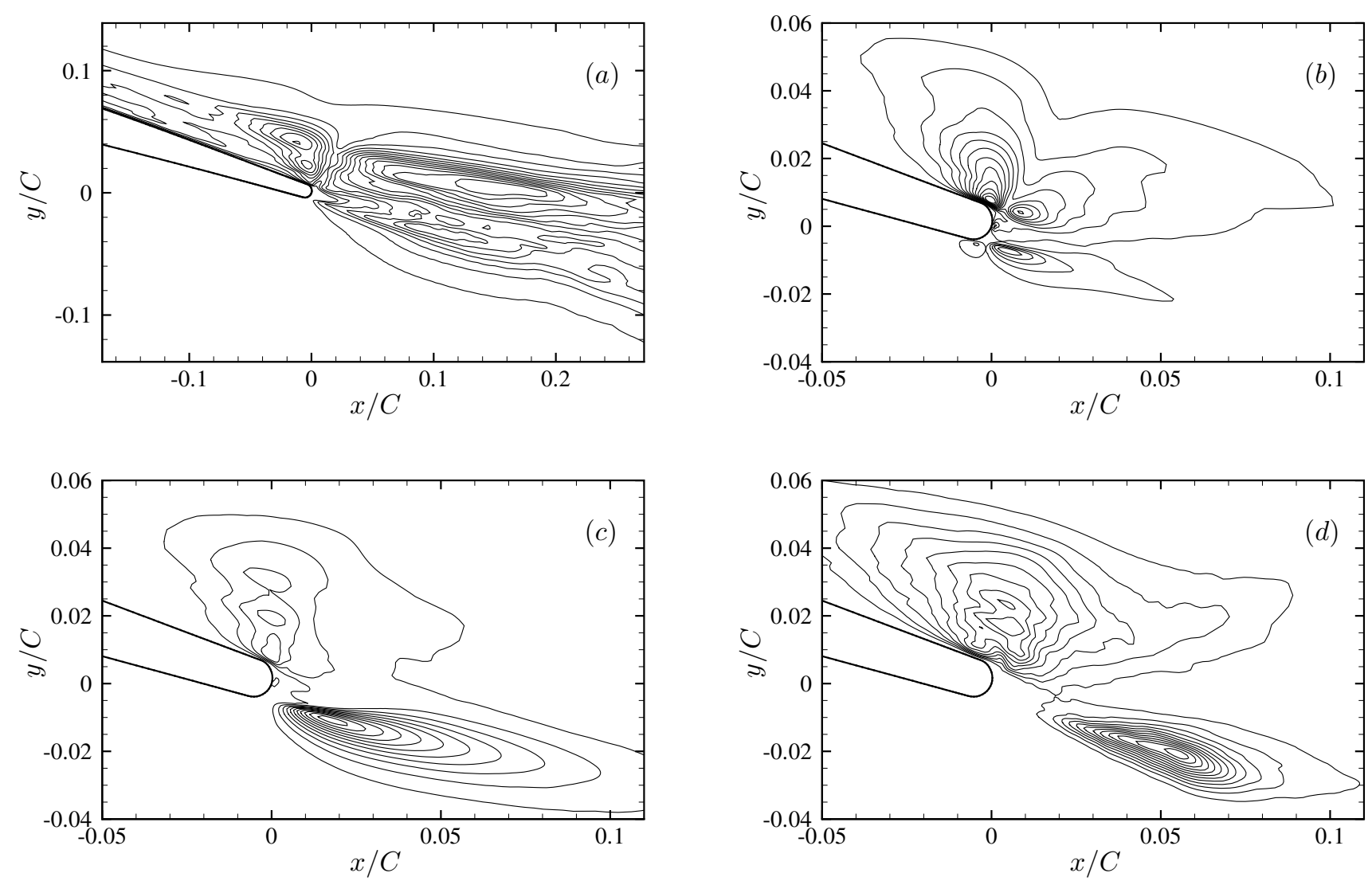

Figure 6: Contours of the magnitude of the source term $\left|-2 \widehat{u_{r} u_{\theta}}\right| / U_{0}^{2}$ for 3 different frequencies : (a) and (b) $446.9 \mathrm{~Hz}$, (c) $1787.8 \mathrm{~Hz}$ and (d) $7151.2 \mathrm{~Hz}$. Sources on Fig. (b) to (c) are multiplied by the factor $r_{0}^{-3 / 2}$.

is then used in Eq. (2) to obtain the far-field sound, and the total radiated sound is computed by ensemble averaging. In order to obtain the sound for the complete experimental airfoil span, the sound spectrum obtained from the previous step is mutiplied by the ratio between the experimental and and numerical span length : $\Phi_{p a}^{\text {tot }}=\left(L_{\text {exp }} / L_{n u m}\right) \Phi_{p a}$. This assumption is valid only if source regions separated by the computational box size radiate in a statistically independent manner, which is the case in the present computations [10].

The determination of the volume of integration $V$ in Eq. (3) is an important issue since it determines the convergence of the integral and the possible boundary impedance problems of the volume integral. The convergence of the integral is illustrated in Fig. 5 for 3 different frequencies in function of the distance of the boundaries of a rectangular box around the trailing edge, in each direction (right/top/left/bottom). The convergence of the integral is mainly depending on the frequency, where, as expected, a larger domain of integration is required to achieve the convergence at low frequencies. Furthermore, the size of the integration domain in the streamwise direction is the most important parameter to reach convergence while small distance from trailing edge could be considered (below $0.2 C$ ) in the other directions.

Figure 6 shows the magnitude of the Reynolds shearstress source term, $\left|-2 \widehat{u_{r} u_{\theta}}\right| / U_{0}^{2}$, in the trailing edge region for three different frequencies. The other componenents of the source term behaves in a similar manner. The source magnitudes are averaged with 15 segments

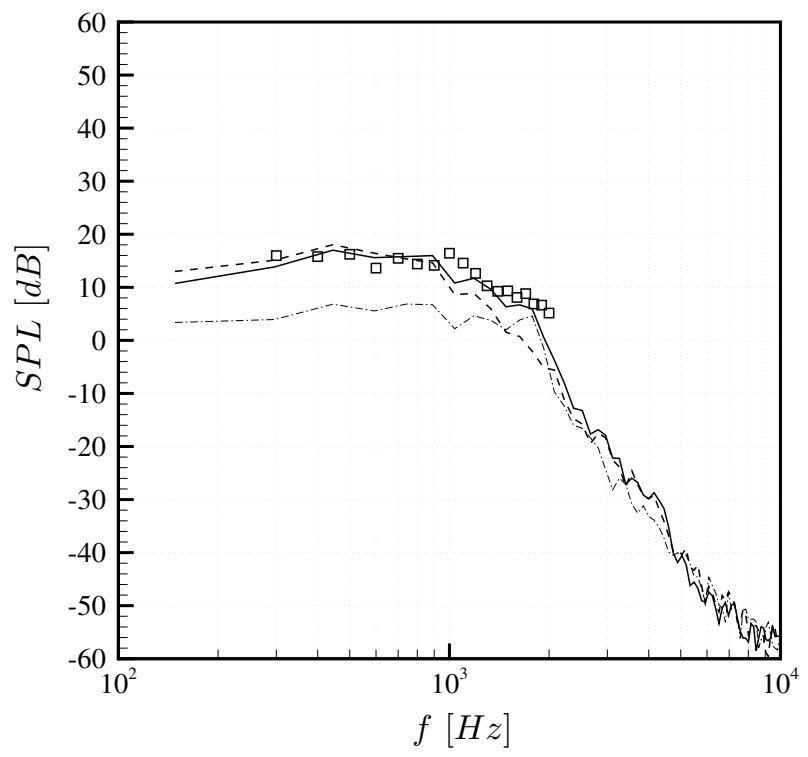

Figure 7: Far field acoustic spectra in the mid span plane above the airfoil $\left(\theta=90^{\circ}\right)$ at $R=2 \mathrm{~m}$ from the trailing edge. Contribution of (dash) the suction side and (dash-dot) the pressure side of the source integral to (plain) the total sound pressure level obtained using

Ffowcs-Williams and Hall's analogy. (square) Experiments

and $50 \%$ overlap, and in the spanwise direction. Note that Fig. 6(a) shows the magnitude of the source term while in Figs. 6(b) to (c), the source terms are weighted, 
as in integral (3), by the factor $r_{0}^{-3 / 2}$. The noise sources are then spread in the whole wall shear layer and wake in the case of Fig. 6(a) while the effective noise source, much more concentrated around the trailing edge, are represented in the other figures. At low frequencies, the sources, related to the large scales unsteady flow structures, are mainly concentrated around the trailing edge, where the largest values are found coming from the attached turbulent boundary layer on the suction side of the airfoil. Smaller values are emanating from the vortex shedding due to the pressure side laminar boundary layer. For the particular frequencies below $f=2000 \mathrm{~Hz}$, the sources of sound, appearing in a large region in the wake, are mainly coming from the vortex shedding. Finally, for the high frequencies, the importance of the source coming from the upper part (suction side) of the wake and the lower part (pressure side) are equivalent.

To confirm this analysis, the volume source of integration in eq. (3) is cut in two parts, separating the lower and upper part of the flow, along a line extending the airfoil chord and having then the same angle than the airfoil angle of attack. The far field noise is then computed for both parts and the total domain, the results being shown in Fig. 3 and compared to experiments. The noise spectrum in the frequency range below $1 \mathrm{kHz}$ is related to the flow structures coming from the turbulent boundary layer on the suction side of the airfoil. Between $1 \mathrm{kHz}$ and $2 \mathrm{kHz}$, the vortex shedding noise contributes in a significant manner to the total farfield sound and is even dominant around $2 \mathrm{kHz}$. In the high frequency part of the noise spectrum, the two phenomena have the same contribution to the total sound. Finally, a good agreament is obtained between the numerical and the experimental sound spectrum.

\section{Conclusion}

The investigation of the flow and the corresponding noise has been studied around a segment of an automotive blade (CD-airfoil) at design conditions, namely a $8^{\circ}$ angle of attack. This study encompasses two aspects, the flow resolution around the profile using the open source OpenFOAM solver and the noise propagation using Ffowcs-Williams and Hall's analogy for trailing edge noise. All along, computational results are compared to experiments taken in the large anechoic chamber from ECL for a Reynolds number $R e_{C}=1.6 \times 10^{5}$. Two mesh refinements in the spanwise direction are studied. It reveals that the improvement of the mesh has a slight influence on the trailing edge spectrum, decreasing the low frequency content to approach experimental results. The noise computation showed a good agreament with experimental measurements and highlighted two different noise mechanisms. The noise coming from the scattering of the suction side boundary layer is mainly appearing at low frequencies while sound produced by vortex shedding from the pressure side boundary layer is present at mid-frequencies. At high frequencies, both mecanisms are present.

\section{Acknowledgments}

J. Christophe is supported by a fellowship from Fonds pour la formation à la Recherche dans l'Industrie et dans
l'Agriculture (FRIA). The participation to the conference has been supported through the FP7 - ECOQUEST project (Grant Agreement no 233541).

\section{References}

[1] Sharland, I.J., "Sources of noise in axial flow fans", J. Sound Vib., 1 (3), 302-322 (1964).

[2] Hubbard, H.H., Shepherd, K.P., "Aeroacoustics of large wind turbines", J. Acoust. Soc. Am. 89, 24952507 (1991).

[3] Pérennès, S., Roger, M., "Aerodynamic noise of a two-dimensional wing with high-lift devices", Fourth AIAA/CEAS Aeroacoustics Conference, Toulouse, France, AIAA Paper 98-2338 (1998).

[4] Wang, M., Moreau, S., Iaccarino, G., Roger, M. "LES prediction of wall pressure spectra on a low speed airfoil", Annual Research Briefs-2004, Center for Turbulence Research, Stanford Univ./NASA Ames (2004).

[5] Moreau, S., Iaccarino, G., Kang, S., Khalighi, Y., Wang, M. "Towards a large eddy simulation of a low speed fan blade", Proceedings of the Summer Program 2004, Center for Turbulence Research, Stanford Univ./NASA Ames (2004).

[6] Moreau, S., Mendonca, F., Qazi, O., Prosser, R., Laurence, D., "Influence of turbulence modeling on airfoil unsteady simulations of broadband noise sources", Eleventh AIAA/CEAS Aeroacoustics Conference, Monterey, Calif, AIAA Paper 2005-2916 (1991).

[7] Moreau, S., Neal, D., Khalighi, Y., Wang, M., Iaccarino, G., "Validation of unstructured-mesh LES of the trailing-edge flow and noise of a controlleddiffusion airfoil" Proceedings of the Summer Program 2006, Center for Turbulence Research, Stanford Univ./NASA Ames (2006).

[8] Moreau, S., Roger, M. "Effect of airfoil aerodynamic loading on trailing edge noise" AIAA J. 43(1), 41-52 (2005).

[9] Moreau, S., Neal, D., Foss, J., "Hot-wire measurements around a controlled diffusion airfoil in an open-jet anechoic wind tunnel", J. Fluid Engineering 28 (4), 699-706 (2006).

[10] Christophe, J., Moreau, S., Anthoine, J. "Trailing edge noise of a Controlled-Diffusion airfoil at Moderate and high angle of attack ", Fifteenth AIAA/CEAS Aeroacoustics Conference, Miami, Florida, AIAA Paper 2009-3196 (2009).

[11] Lighthill, M., "On sound generated aerodynamically: I. General theory", Proc. R. Soc. London Ser. A, 211 (1107), 564-587 (1952).

[12] Curle, N., "The influence of solid boundaries upon aerodynamic sound", Proc. R.Soc. London Ser. A, 231 (1187), 505-514 (1955). 